\title{
ACHADOS VESTIBULARES EM USUÁRIOS DE APARELHO DE AMPLIFICAÇÃO SONORA INDIVIDUAL
}

\author{
Vestibular findings in hearing aid users
}

\author{
Fabiane Paulin (1), Bianca Simone Zeigelboim ${ }^{(2)}$, Karlin Fabianne Klagenberg ${ }^{(3)}$, \\ Marine Raquel Diniz da Rosa ${ }^{(4)}$
}

\section{RESUMO}

Objetivo: verificar os achados vestibulares em pacientes com perda auditiva neurossenssorial usuários de aparelho de amplificação sonora individual. Métodos: vinte pacientes, 11 do sexo feminino e nove do sexo masculino, com idades entre 39 e 85 anos, com perda auditiva neurossenssorial bilateral de grau moderado e severo foram atendidos em uma Instituição de Ensino Superior e submetidos a uma anamnese, inspeção otológica, avaliação audiológica, imitanciometria e ao exame vestibular por meio da vectoeletronistagmografia. Resultados: a) dos 20 pacientes avaliados, 18 $(90 \%)$ apresentaram queixa de zumbido, 15 (75\%) queixa de tontura e oito (40\%) queixa de cefaléia; b) houve predomínio de alteração na prova calórica e no sistema vestibular periférico; c) o resultado do exame vestibular esteve alterado em 14 pacientes (70\%), sendo, oito casos (40\%) de síndrome vestibular periférica irritativa e seis casos $(30 \%)$ de síndrome vestibular periférica deficitária; d) verificou-se diferença significativa entre o resultado do exame vestibular e o tempo de uso do aparelho de amplificação sonora individual; e) dos cinco pacientes que não referiram nenhum sintoma vestibular, quatro $(80 \%)$ apresentaram alteração no exame. Conclusão: ressalta-se a sensibilidade e importância do estudo funcional do sistema do equilíbrio neste tipo de população, uma vez que podem ocorrer alterações na avaliação labiríntica independente da presença de sintomas.

DESCRITORES: Audição; Perda Auditiva; Testes de Função Vestibular; Auxiliares de Audição

\section{INTRODUÇÃO}

De acordo com a Sociedade Brasileira de Otologia e a Organização Mundial de Saúde ', mais de 15 milhões de brasileiros possuem alteração de audição. Este problema ganha muitas vezes contornos preocupantes e poderia ser resolvido por meio

(1) Aluna do Curso de Graduação em Fonoaudiologia da Universidade Tuiuti do Paraná, UTP, Curitiba, PR.

(2) Fonoaudióloga; Professora Adjunto do Curso de Fonoaudiologia; Coordenadora do Programa de Mestrado e Doutorado em Distúrbios da Comunicação da Universidade Tuiuti do Paraná, UTP, Curitiba, PR; Pós-Doutora em Distúrbios da Comunicação Humana pela Universidade Federal de São Paulo.

(3) Fonoaudióloga; Mestre em Distúrbios da Comunicação; Doutoranda em Distúrbios da Comunicação pela Universidade Tuiuti do Paraná, UTP, Curitiba, PR.

(4) Fonoaudióloga; Professora do Curso de Graduação em Fonoaudiologia do Centro Universitário de João Pessoa, Unipê, João Pessoa, PE; Mestre em Distúrbios da Comunicação pela Universidade Tuiuti do Paraná. de tratamento medicamentoso, cirúrgico ou com o uso de aparelhos de amplificação sonora individual (AASI). Apenas $40 \%$ dos portadores de perda auditiva reconhecem a doença. A falta de informação e o preconceito fazem com que a maioria dos indivíduos demore, em média, seis anos para procurar auxílio.

A deficiência auditiva é um dos fatores mais incapacitantes, delimitando ou até mesmo impedindo o portador de tal deficiência de desempenhar plenamente suas funções na sociedade. Portar deficiência auditiva adquirida abrange muito mais do que o simples fato de não ouvir bem, pois deve levar-se em consideração os problemas causados pelas desvantagens oriundas dessa deficiência em diversas situações de vida diária, tais como: diminuição da percepção de fala em ambientes ruidosos, aumento do volume da televisão e rádio, dificuldade para compreender em cinema, teatros, cultos em igrejas, sinais sonoros de alerta, música e sons ambientais. Os AASIs são instrumentos importantes em casos 
de perdas auditivas não passíveis de tratamento médico ou cirúrgico ${ }^{2}$.

Devido à proximidade das estruturas responsáveis pelas funções auditiva e vestibular, é comum encontrar alterações associadas em ambos os sistemas; entretanto, a queixa de desequilíbrio nem sempre é observada ${ }^{3}$.

A capacidade de ouvir é na verdade uma característica secundária. A primeira responsabilidade do órgão auditivo é a manutenção do equilíbrio. $O$ aparelho vestibular é o órgão que detecta as sensações de equilíbrio corporal, de fundamental importância no relacionamento espacial do organismo com o ambiente. Assim, para que isso ocorra, é necessária uma perfeita integração entre a visão, a sensibilidade proprioceptiva e os sistemas auditivo e vestibular ${ }^{4}$. Estudo ${ }^{5}$ indica que todo paciente com diagnóstico de perda auditiva neurossensorial, independente da idade ou sexo, deve submeter-se ao exame vestibular, mesmo na ausência de vertigem e outras tonturas. Os autores referem que, confirmada a perda auditiva do tipo neurossensorial, torna-se obrigatória a realização do exame vestibular, mesmo na ausência de sintomas, como a tontura, pois pode haver uma alteração vestibular importante, geralmente assintomática, provavelmente pela instalação concomitante de mecanismos de auto-compensação, diante da lenta evolução do distúrbio.

Em casos de surdez de graus severo e profundo é significantemente elevada a incidência de achados eletronistagmográficos anormais, independente do diagnóstico topográfico ser periférico, central ou misto ${ }^{6}$. Desta forma, o objetivo deste estudo foi verificar os achados vestibulares em pacientes com perda auditiva neurossensorial usuários de AASI.

\section{MÉTODOS}

Avaliaram-se 20 pacientes, sendo 11 (55\%) do sexo feminino e nove (45\%) do sexo masculino, com idades entre 39 e 85 anos, média de 63,35 anos (desvio padrão 12,14). Incluíram-se na pesquisa portadores de perda auditiva neurossenssorial bilateral de grau moderado e severo, usuários de AASI, sendo, sete com uso unilateral e 13 com uso bilateral, atendidos em uma Instituição de Ensino Superior e excluíram-se pacientes portadores de alterações neurológicas, psicológicas, visuais, musculoesqueléticas importantes e outras que impossibilitavam a realização dos exames.

Os pacientes foram submetidos aos seguintes procedimentos:

Anamnese: Aplicou-se um questionário com ênfase aos sinais e sintomas otoneurológicos, antecedentes pessoais e familiares.
Avaliação Otorrinolaringológica: Realizada com o objetivo de excluir qualquer alteração que pudesse interferir no exame.

Avaliação Audiológica: A audiometria tonal limiar convencional foi realizada em cabina acústica padronizada segundo ANSI S31 (1989) com audiômetro Interacoustics AC 40, com fones TDH 39P e com limiares em dB NA. Aplicaram-se as classificações de Davis e Silverman ${ }^{7}$ e Silman e Silverman ${ }^{8}$ para caracterização do grau e tipo de perda auditiva.

Medidas de Imitância Acústica: Este procedimento foi realizado para avaliar a integridade do sistema tímpano-ossicular por meio da curva timpanométrica e da pesquisa do reflexo acústico. $\mathrm{O}$ equipamento utilizado foi o impedanciômetro Interacoustics AZ-7 e fones TDH 39P.

Avaliação Vestibular: Os pacientes foram submetidos às seguintes provas que compõem 0 exame vestibular:

\section{Sem registro}

Pesquisaram-se o nistagmo e a vertigem de posição/posicionamento e os nistagmos espontâneo e semi-espontâneo com os olhos abertos, no olhar frontal e a 30 graus de desvio do olhar nos quatro pontos cardinais.

\section{Com registro}

Para a realização da vectoeletronistagmografia (VENG) utilizou-se um aparelho termossensível, com três canais de registro, da marca Berger, modelo VN316. Após a limpeza da pele das regiões periorbitárias com álcool, colocaram-se em cada paciente, fixados com pasta eletrolítica, um eletródio ativo no ângulo lateral de cada olho e na linha média frontal, formando um triângulo isósceles, que permitiu a identificação dos movimentos oculares horizontais, verticais e oblíquos. Este tipo de VENG permitiu obter medidas mais precisas da velocidade angular da componente lenta (VACL) do nistagmo (correção vestibular).

Utilizou-se uma cadeira rotatória pendular decrescente da marca Ferrante, um estimulador visual modelo EV VEC, e um otocalorímetro a ar modelo NGR 05, ambos da marca Neurograff. Realizaram-se as seguintes provas oculares e labirínticas à VENG, segundo os critérios propostos por Mangabeira-Albernaz, Ganança e Pontes ${ }^{9}$.

- Calibração dos movimentos oculares: nesta etapa do exame avaliou-se a regularidade do traçado, o que torna as pesquisas comparáveis entre si.

- Pesquisa dos nistagmos espontâneo (olhos abertos e fechados) e semi-espontâneo (olhos abertos): nesse registro avaliaram-se a 
ocorrência, direção, efeito inibidor da fixação ocular (EIFO) e o valor da VACL máxima do nistagmo.

- Pesquisa do rastreio pendular: avaliaram-se a ocorrência e o tipo de curva.

- Pesquisa do nistagmo optocinético, à velocidade de $60^{\circ}$ por segundo, nos sentidos anti-horário e horário, na direção horizontal. Avaliaram-se a ocorrência, direção, VACL máxima às movimentações anti-horária e horária do nistagmo.

- Pesquisa dos nistagmos pré e pós-rotatórios à prova rotatória pendular decrescente, com estimulação dos ductos semicirculares laterais, anteriores e posteriores. Para a estimulação dos ductos semicirculares laterais (horizontais) a cabeça foi fletida $30^{\circ}$ para frente. $\mathrm{Na}$ etapa seguinte, para a sensibilização dos ductos semicirculares anteriores e posteriores (verticais) o posicionamento da cabeça foi de $60^{\circ}$ para trás e $45^{\circ}$ à direita e, a seguir, $60^{\circ}$ para trás e $45^{\circ}$ à esquerda, respectivamente. Observaram-se a ocorrência, direção, frequência às rotações antihorária e horária do nistagmo.

- Pesquisa dos nistagmos pré e pós-calóricos realizada com o paciente posicionado de forma que a cabeça e o tronco estivessem inclinados $60^{\circ}$ para trás, para estimulação adequada dos ductos semicirculares laterais. O tempo de irrigação de cada orelha com ar a $42^{\circ} \mathrm{C}$ e $18^{\circ} \mathrm{C}$ durou 80 s para cada temperatura e as respostas foram registradas com os olhos fechados e, a seguir, com os olhos abertos para a observação do EIFO. Nesta avaliação verificaram-se a direção, os valores absolutos da VACL e o cálculo das relações da preponderância direcional do nistagmo (PDN) e predomínio labiríntico (PL) do nistagmo pós-calórico.

Este estudo foi aprovado pelo Comitê de Ética Institucional, parecer no. 099/2006, e autorizado pelos pacientes por meio da assinatura do Termo de Consentimento Livre e Esclarecido.

Para a análise dos resultados utilizaram-se métodos de estatística descritiva (tabelas de frequências) e o teste de Fischer, ao nível de significância de 0,05 (5\%).

\section{RESULTADOS}

As queixas otoneurológicas mais referidas na anamnese foram: zumbido (90\%), tontura (75\%) e cefaléia (40\%), conforme descrito na Tabela 1.

Em relação aos outros achados clínicos de maior ocorrência, estavam presentes, a hipertensão arterial sistêmica (30\%), o colesterol total elevado $(20 \%)$ e os distúrbios reumáticos (15\%).
A Tabela 2 mostra que o exame vestibular esteve alterado em $70 \%$ e normal em $30 \%$ dos pacientes. Das alterações evidenciadas quatro pacientes (20\%) apresentaram síndrome vestibular periférica deficitária unilateral, três (15\%) apresentaram síndrome vestibular periférica irritativa bilateral, três $(15 \%)$ apresentaram síndrome vestibular periférica irritativa unilateral, dois (10\%) apresentaram síndrome vestibular periférica deficitária bilateral e dois $(10 \%)$ apresentaram síndrome vestibular periférica irritativa.

Aplicou-se o Teste de Fisher para comparação do resultado do exame com o sexo, idade e queixas apresentadas. Os resultados não foram significantes para o sexo $(p=0,6424)$, para a idade $(p=1,0000)$ e para as queixas apresentadas $(p=1,0000)$.

O tempo de uso de AASI nos 20 pacientes resultou em um tempo médio de 2,35 anos, mínimo de um ano e máximo de cinco anos, com um desvio padrão de 1,69.

Dos 20 pacientes avaliados, sete (35\%) usam AASI do tipo monoaural e $13(65 \%)$ do tipo binaural.

Aplicou-se o Teste de Fisher para comparação do tipo e tempo de uso de AASI com o resultado do exame vestibular e queixas apresentadas. Verificou-se que ocorreu diferença significante entre o resultado do exame vestibular e o tempo de uso de AASI $(p=0,0419)$ conforme denota a Tabela 3.

Aplicou-se o Teste de Fisher para a comparação do resultado do exame vestibular com os sinais e sintomas otoneurológicos e os outros achados de maiores ocorrências. Verificou-se não existir diferença significante conforme demonstra a Tabela 4.

Para a comparação do tipo de AASI com o resultado do exame vestibular, sinais e sintomas otoneurológicos e outros achados de maiores ocorrências, aplicou-se o Teste de Fisher e não foram encontradas diferenças significantes conforme mostra a Tabela 5.

\section{DISCUSSÃO}

$\mathrm{Na}$ presente pesquisa houve predomínio do sexo feminino, concordando com diversos autores ${ }^{10,11}$. Alguns trabalhos ${ }^{12,13}$ relatam que a tontura é mais frequente no sexo feminino, na proporção 2:1. Acredita-se que o sexo feminino apresenta maior predisposição orgânica às disfunções vestibulares devido à sua intrínseca variação hormonal e aos distúrbios metabólicos frequentemente encontrados na mulher. Outro fator relevante em nosso meio é a maior preocupação em procurar orientação médica apresentada pelas mulheres em relação aos homens. 
Tabela 1 - Frequência das manifestações clínicas referidas na anamnese em 20 pacientes usuários de AASI

\begin{tabular}{lcc}
\hline SINAIS E SINTOMAS & N & FREQUENCIA (\%) \\
\hline Zumbido & 18 & 90 \\
Tontura & 15 & 75 \\
Cefaléia & 8 & 40 \\
Náusea & 5 & 25 \\
Insônia & 4 & 20 \\
Dificuldade ou dor ao movimento do pescoço & 4 & 20 \\
Desconforto a sons intensos & 4 & 15 \\
Depressão & 3 & 15 \\
Estalo no pescoço & 3 & 15 \\
Ansiedade & 3 & 15 \\
Visão borrada & 3 & 10 \\
Dificuldade para falar & 2 & 10 \\
Formigamento de extremidades & 2 & 5 \\
Fadiga & 1 & 5 \\
Visão dupla & 1 & 5 \\
Alteração voz & 1 & 5 \\
Cegueira temporária & 1 & 5 \\
Pesadelo & 1 & 5 \\
Desmaio & 1 & 5 \\
Alteração da sensibilidade & 1 & 5 \\
Formigamento da face & 1 & 5 \\
Incoordenação motora & 1 & 5 \\
Alteração do olfato & 1 & 5 \\
Dificuldade para engolir & 1 & \\
\hline
\end{tabular}

Tabela 2 - Resultado final do exame vestibular

\begin{tabular}{lc}
\hline RESULTADO & NÚMERO DE CASOS (\%) \\
\hline Normorreflexia & $6(30 \%)$ \\
S.V.P.D.U. & $4(20 \%)$ \\
S.V.P.I.B. & $3(15 \%)$ \\
S.V.P.I.U. & $3(15 \%)$ \\
S.V.P.D.B. & $2(10 \%)$ \\
S.V.P.I. & $2(10 \%)$ \\
\hline
\end{tabular}

Em relação às principais manifestações clínicas referidas na anamnese, observou-se: zumbido $(90 \%)$, tontura $(75 \%)$, cefaléia $(40 \%)$, náuseas $(25 \%)$, insônia $(20 \%)$, dificuldade ou dor para movimentar o pescoço $(20 \%)$ e desconforto a sons intensos $(20 \%)$, o que se assemelham a outros estudos que investigaram a tontura ${ }^{14}$.

Em um estudo realizado com 3.701 pacientes submetidos a exame clínico e vestibular por meio da VENG ${ }^{15}$, observou-se a presença de manifes- tações clínicas como tontura, zumbido, sintomas neurovegetativos e alterações auditivas como sendo as queixas mais frequentes e estando presente na maioria dos casos avaliados. Apenas $8 \%$ da população estudada não se queixaram de tontura, as alterações auditivas estavam presentes em $68 \%$, manifestações neurovegetativas em $67 \%$ e zumbido em $59 \%$ dos casos. O alto índice de queixas relacionadas ao zumbido $90 \%$ concomitantes as alterações auditivas na presente pesquisa concorda com estudos ${ }^{16}$ que, ao avaliar 51 pacientes, encontraram este sintoma em $64 \%$ dos casos.

A tontura, presente em $75 \%$ dos pacientes, corrobora outros estudos, que ao realizarem avaliação vestibular em idosos, encontraram também a prevalência desse sintoma ${ }^{10}$.

Alterações do equilíbrio podem vir acompanhadas de sintomatologia neurovegetativa. No presente estudo, dos 15 pacientes com queixa de tontura, $33 \%$ relataram sintomas neurovegetativos, principalmente náuseas. Existe um consenso na literatura de que a ocorrência destes sintomas é considerada frequente na população geriátrica. 
Tabela 3 - Comparação do tipo de AASI com o resultado do exame vestibular, queixas apresentadas e tempo de uso

\begin{tabular}{|c|c|c|c|}
\hline \multirow{2}{*}{$\begin{array}{l}\text { Prótese } \\
\text { Tipo } \\
\end{array}$} & \multicolumn{2}{|c|}{ Exame } & \multirow[t]{2}{*}{$\mathbf{P}$} \\
\hline & Alterado & Normal & \\
\hline Monoaural & 3 & 4 & \\
\hline Binaural & 11 & 2 & 0,1219 \\
\hline \multirow[t]{2}{*}{ Tipo } & \multicolumn{2}{|c|}{ Queixa } & \multirow[t]{2}{*}{$P$} \\
\hline & Presença & Ausência & \\
\hline Monoaural & 2 & 5 & \\
\hline Binaural & 3 & 10 & 0,5942 \\
\hline \multirow{2}{*}{ Tipo } & \multicolumn{2}{|c|}{ Tempo } & \multirow[t]{2}{*}{$\mathrm{P}$} \\
\hline & 1 a 2 anos & 3 a 5 anos & \\
\hline Monoaural & 5 & 2 & \\
\hline Binaural & 7 & 6 & 0,6424 \\
\hline \multirow[t]{2}{*}{ Tempo de uso } & \multicolumn{2}{|c|}{ Exame } & \multirow[t]{2}{*}{$P$} \\
\hline & Alterado & Normal & \\
\hline 1 a 2 anos & 6 & 6 & \\
\hline 3 a 5 anos & 8 & 0 & ${ }^{*} 0,0419$ \\
\hline \multirow{2}{*}{ Tempo de uso } & \multicolumn{2}{|c|}{ Queixa } & \multirow{2}{*}{$P$} \\
\hline & Presença & Ausência & \\
\hline 1 a 2 anos & 11 & 1 & \\
\hline 3 a 5 anos & 4 & 4 & 0,1089 \\
\hline
\end{tabular}

Tabela 4 - Comparação dos resultados do exame vestibular com os sinais e sintomas otoneurológicos e os outros achados de maior ocorrência

\begin{tabular}{|c|c|c|c|c|}
\hline \multicolumn{5}{|c|}{ SINAIS E SINTOMAS OTONEUROLÓGICOS } \\
\hline & & \multicolumn{2}{|c|}{ Resultado do exame } & $P$ \\
\hline \multirow{3}{*}{ Zumbido } & & Normal & Alterado & \\
\hline & Ausência & 0 & 3 & \\
\hline & Presença & 6 & 11 & 0,5221 \\
\hline \multirow{3}{*}{ Tontura } & & Normal & Alterado & \\
\hline & Ausência & 1 & 4 & \\
\hline & Presença & 5 & 10 & 1,0000 \\
\hline \multirow{3}{*}{ Dor de cabeça } & & Normal & Alterado & \\
\hline & Ausência & 5 & 7 & \\
\hline & Presença & 1 & 7 & 0,3246 \\
\hline \multirow{3}{*}{ Náuseas } & & Normal & Alterado & \\
\hline & Ausência & 6 & 9 & \\
\hline & Presença & 0 & 5 & 0,2604 \\
\hline \multicolumn{5}{|c|}{ OUTROS ACHADOS } \\
\hline & & \multicolumn{2}{|c|}{ Resultado do exame } & $P$ \\
\hline \multirow{3}{*}{ Hipertensão arterial } & & Normal & Alterado & \\
\hline & Ausência & 4 & 10 & \\
\hline & Presença & 2 & 4 & 1,0000 \\
\hline \multirow{3}{*}{$\begin{array}{l}\text { Colesterol total } \\
\text { elevado }\end{array}$} & & Normal & Alterado & \\
\hline & Ausência & 4 & 12 & \\
\hline & Presença & 2 & 2 & \\
\hline \multirow{3}{*}{$\begin{array}{l}\text { Distúrbios } \\
\text { reumáticos }\end{array}$} & & Normal & Alterado & 0,5492 \\
\hline & Ausência & 5 & 12 & \\
\hline & Presença & 1 & 2 & 1,0000 \\
\hline
\end{tabular}


Tabela 5 - Comparação do tipo de AASI com relação ao resultado do exame vestibular, sinais e sintomas otoneurológicos e outros achados de maiores ocorrências

\begin{tabular}{|c|c|c|c|c|}
\hline \multicolumn{5}{|c|}{ SINAIS E SINTOMAS OTONEUROLÓGICOS } \\
\hline & & \multicolumn{2}{|c|}{ Tipo da prótese } & $\mathrm{P}$ \\
\hline \multirow{3}{*}{ Zumbido } & & Monoaural & Binaural & \\
\hline & Ausência & 1 & 2 & \\
\hline & Presença & 6 & 11 & 0,7285 \\
\hline \multirow{3}{*}{ Tontura } & & Monoaural & Binaural & \\
\hline & Ausência & 2 & 3 & \\
\hline & Presença & 5 & 10 & 1,0000 \\
\hline \multirow{3}{*}{ Dor de cabeça } & & Monoaural & Binaural & \\
\hline & Ausência & 4 & 8 & \\
\hline & Presença & 3 & 5 & 1,0000 \\
\hline \multirow{3}{*}{ Náuseas } & & Monoaural & Binaural & \\
\hline & Ausência & 6 & 9 & \\
\hline & Presença & 1 & 4 & 0,6126 \\
\hline \multicolumn{5}{|c|}{ OUTROS ACHADOS } \\
\hline & & \multicolumn{2}{|c|}{ Tipo da prótese } & $\mathrm{P}$ \\
\hline \multirow{3}{*}{ Hipertensão arterial } & & Monoaural & Binaural & \\
\hline & Ausência & 7 & 7 & \\
\hline & Presença & 0 & 6 & 0,0515 \\
\hline \multirow{3}{*}{$\begin{array}{l}\text { Colesterol total } \\
\text { elevado }\end{array}$} & & Monoaural & Binaural & \\
\hline & Ausência & 4 & 12 & \\
\hline & Presença & 3 & 1 & 0,1011 \\
\hline \multirow{3}{*}{$\begin{array}{l}\text { Distúrbios } \\
\text { reumáticos }\end{array}$} & & Monoaural & Binaural & \\
\hline & Ausência & 5 & 12 & \\
\hline & Presença & 2 & 1 & 0,2702 \\
\hline
\end{tabular}

Observa-se nesta população um aumento crescente dos distúrbios das funções sensoriais, da integração das informações periféricas e centrais, bem como a senescência dos sistemas neuromusculares e da função esquelética ${ }^{11,17}$.

Em relação aos outros achados de maior ocorrência, observou-se nos pacientes, 30\% com hipertensão arterial, $20 \%$ com colesterol total elevado e $15 \%$ com distúrbios reumáticos. Estes achados foram concordantes com o autor ${ }^{3}$ que, referiu que os principais distúrbios circulatórios que podem causar comprometimento periférico ou central nos sistemas auditivo e vestibular são hiper ou hipotensão arterial, insuficiência cardíaca, aumento do colesterol, arritmias, entre outros.

Houve alta prevalência de exames vestibulares alterados $(70 \%)$, concordante com um estudo ${ }^{10}$, que ao avaliar 34 pacientes com tontura ou não, com idade média de 72 anos, portadores de perda auditiva neurossenssorial (97\%), encontrou $68 \%$ de alterações no exame vestibular. Algumas causas de deficiência auditiva neurossenssorial podem envolver o labirinto, provavelmente destruindo o ouvido interno, como por exemplo, meningite, traumatismos, hemorragias e síndromes genéticas.

Ao pesquisar 26 crianças portadoras de perda auditiva, o autor ${ }^{18}$ encontrou alteração no exame vestibular com predomínio da síndrome vestibular periférica em $96 \%$ dos pacientes, independente do grau de perda auditiva, o que concorda com outro estudo ${ }^{15}$ que verificou a prevalência das alterações periféricas $36 \%$ ao analisar 3701 prontuários de pacientes submetidos ao exame vestibular com idade de dois a mais de 60 anos.

Não foram observados sinais de alterações centrais ao exame vestibular. Este achado é semeIhante ao dos autores consultados ${ }^{10,11,19,20}$, que não encontraram à avaliação vestibular em indivíduos com perdas auditivas neurossenssoriais sinas de comprometimento da via vestibular central.

Neste estudo, foram observados $40 \%$ de síndrome vestibular periférica do tipo irritativa e $30 \%$ de síndrome vestibular periférica do tipo deficitária. Esses resultados assemelham-se ao autor ${ }^{19}$ que encontrou a prevalência das síndromes irritativas em $41 \%$ dos indivíduos. 
Estudos ${ }^{21}$ afirmam que, fisiologicamente, a síndrome irritativa causada por hiperreatividade se dá devido à menor atuação dos mecanismos inibidores do sistema vestibular de provável origem cerebelar, em consequência à perda das células inibitórias de Purkinge. Os outros autores ${ }^{22,23}$ referem que ocorrem perdas de células ciliadas de cristais e máculas com a idade, juntamente com o declínio no número de células nervosas do gânglio de Scarpa e degeneração de otocônias, que se fragmentam e desaparecem. A somatória de todas essas modificações resulta em diminuição do input das células ciliadas. Outro fator a ser considerado é a diminuição do fluxo sanguíneo labiríntico e a progressiva depressão da estabilidade neural com o aumento da idade, contribuindo para a hiporreatividade do sistema vestibular.

Ao comparar o tipo e tempo de uso dos AASI com o resultado do exame vestibular e queixas apresentadas, observou-se resultado significante em pacientes que faziam uso de AASI de três a cinco anos. Não se encontrou na literatura pesquisada dados que justificassem esses achados.

O ser humano necessita manter o equilíbrio corporal para exercer adequadamente suas atividades profissionais e de lazer. Autores citam uma alta prevalência de alterações vestibulares e auditivas, o que justifica a necessidade destes pacientes serem sistematicamente avaliados 10,24. Recomenda-se incluir, portanto, a avaliação do sistema vestibular como uma importante ferramenta na bateria de testes que um indivíduo, candidato ao uso de AASI deve submeter-se, tendo em vista que, pode haver alteração vestibular mesmo na ausência de sintomas labirínticos.

\section{CONCLUSÃO}

Houve alteração no exame vestibular em $70 \%$ dos pacientes com prevalência do comprometimento do sistema vestibular periférico e das síndromes vestibulares periféricas irritativas.

\begin{abstract}
Purpose: to check vestibular findings in patients with sensoneural hearing loss, hearing aid users. Methods: 20 patients (eleven females and nine males) aging from 39 to 85-year-old with bilateral sensorineural hearing loss, from moderate to severe degrees, were attended in a higher education institution evaluated by medical history, otological inspections, complete basic conventional audiological evaluations, acoustic impedance tests and vectoeletronystagmography. Results: a) from the 20 evaluated patients, $18(90 \%)$ showed tinnitus complaint, $15(75 \%)$ dizziness complaint and eight $(40 \%)$ headache complaint; b) There was a prevalence of alterations in the caloric test and in the peripheral vestibular system; c) the results of the vestibular exam showed alterations in 14 patients $(70 \%)$ being, eight cases $(40 \%)$ of peripheral vestibular irritative syndromes and six cases $(30 \%)$ of peripheral vestibular deficiency syndromes; d) we verified significant difference between the vestibular exam results and how long the patient had use the hearing aid; e) from the five patients that did not related vestibular symptoms four $(80 \%)$ showed alterations in the vestibular exam. Conclusion: the importance and sensibility of the functional study regarding the balance system in this type of population must be emphasized because same disorders may occur in the vestibular exam in spite of symptom presence.
\end{abstract}

KEYWORDS: Hearing; Hearing Loss; Vestibular Function Tests; Hearing Aids

\section{REFERÊNCIAS}

1. Sociedade Brasileira de Otologia. Disponível em URL: http://<www.sbotologia.com.br>. Acesso em Jun 2006.

2. Russo ICP, Almeida K. O processo de reabilitação audiológica do deficiente auditivo idoso. In: Marchesan IQ, Bolaffi C, Gomes ICD, Zorzi JL.
Tópicos de fonoaudiologia. São Paulo: Lovise; 1995. p. 430-47.

3. Caovilla HH, Ganança MM, Munhoz MSL, Silva, MLG, Ganança FF. Presbivertigem, presbiataxia, presbizumbido e presbiacusia. In: Silva MLG, Munhoz MSL, Ganança MM, Caovilla HH. Quadros clínicos otoneurológicos mais comuns. São Paulo: Atheneu; 2000. p.153-8. 
4. Northern JL, Downs MP. Audição em crianças. 3. ed. São Paulo: Manole; 1989.

5. Zeigelboim BS, Jurkiewicz AL, Ribeiro SBA, Martins-Bassetto J, Klagenberg KF. Achados vestibulococleares em indivíduos com zumbido sem queixa de tontura. Arq Int Otorrinolaringol. $2005 \mathrm{Jul} /$ Set; 9(3):196-201.

6. Fernandes JR, Ganança CF. Avaliação vestibular analógica e computadorizada em pacientes com distúrbios de origem metabólica. Arq Int Otorrinolaringol. $2004 \mathrm{Jul} / \mathrm{Set} ;$ 8(3):208-14.

7. Davis H, Silverman SR. Auditory test hearing aids. In: Davis H, Silverman RS. Hearing and deafness. 3. ed. New York; Holt, Rinehart \& Wilson; 1970.

8. Silman S, Silverman CA. Basic audiologic testing. In: Silman S, Silverman CA, Auditory diagnosis, principles and applications. San Diego: Singular Publishing Group; 1997. p. 38-58.

9. Mangabeira-Albernaz PL, Ganança MM, Pontes PAL. Modelo operacional do aparelho vestibular. In: Mangabeira-Albernaz PL, Ganança MM. Vertigem. 2. ed. São Paulo: Moderna; 1976. p. 29-36.

10. Gushikem P, Caovilla HH, Ganança, MM. Avaliação otoneurológica em idosos com tontura. Acta Awho. 2003 Jan/Mar; 21(1):1-25.

11. Zanardini $\mathrm{FH}$, Zeigelboim BS, Jurkiewicz AL, Marques JM, Martins-Bassetto J. Reabilitação vestibular em idosos com tontura. Pró-Fono. 2007 Abr/Jun; 19(2):177-84.

12. Campos $\mathrm{CAH}$. Principais quadros clínicos no adulto e no idoso. In: Ganança MM. Vertigem tem cura? São Paulo: Lemos Editorial; 1998. p. 49-57. 13. Gazzola JM, Ganança FF, Aratani MC, Perracini MR, Ganança MM. Caracterização clínica de idosos com disfunção vestibular crônica. Rev Bras Otorrinolaringol. $2006 \mathrm{Jul} / \mathrm{Ago}$; 72(4):515-22.

14. Ruwer SL, Rossi AG, Simon LF. Equilíbrio no idoso. Rev Bras Otorrinolaringol. 2005 Mai/Jun; 71(3):298-303.
15. Lourenço EA, Lopes KC, Pontes Junior A, Oliveira MH, Umemura A, Vargas AL. Distribuição dos achados otoneurológicos em pacientes com disfunção vestíbulo-coclear. Rev Bras Otorrinolaringol. 2005 Mai/Jun; 71(3):288-96.

16. Teixeira AR, Freitas CR, Millão LF, Gonçalves AK, Becker Junior B, Vieira AF et al. Relação entre deficiência auditiva, idade, gênero e qualidade de vida de idosos. Arq Int Otorrinolaringol. 2008 Jan/ Mar; 12(1):62-70.

17. Simoceli L, Bittar RMS, Bottino MA, Bento RF. Perfil diagnóstico do idoso portador de desequilíbrio corporal: resultados preliminares. Rev Bras Otorrinolaringol. 2003 Nov/Dez; 69(6):772-7.

18. Lisboa TR, Jurkiewicz AL, Zeigelboim BS, Martins-Basseto J, Klagenberg KF. Achados vestibulares em crianças deficientes auditivas. Arq Int Otorrinolaringol. 2005 Out/Dez; 9(4):271-9.

19. Mota PHM, Franco ES, Pinto ESM, Arieta AM. Estudo de equilíbrio no idoso por meio da eletronistagmografia. Acta Awho. $2006 \mathrm{Jul} / \mathrm{Set}$; 24(3):163-9.

20. Mariotto LDF, Alvarenga KF, Costa Filho OA. Avaliação vestibular na perda auditiva neurossensorial unilateral: estudo vectoelectronistagmográfico. Dist Comun. 2006 Abr; 18(1):27-38.

21. Cahali RB, Reis FO, Romano FR, Bittar RMS, Formigoni LG. Eletronistagmografia do paciente idoso: avaliação retrospectiva de 35 casos. Arq Otorrinolaringol. $2000 \mathrm{Abr} / \mathrm{Jun} ; 4$ 4(2):75-80.

22. Gulya A. Neuroanatomy and physiology of the vestibular system relavant to disequilibrium in the elderly. Ear Nose Throat J. 1989; 68:915-23.

23. Belal A, Glorig A. Disequilibrium of aging (presbyastasis). J Laryngol Otol. 1986; 100: 1037-41.

24. Imbaud Genieys S. [Vertigo, dizziness and falls in the elderly]. Ann Otolaryngol Chir Cervicofac. 2007 Sep; 124(4):189-96.

RECEBIDO EM: 03/06/2008

ACEITO EM: 03/12/2008

Endereço para Correspondência:

Bianca Simone Zeigelboim

Rua Gutemberg, 99 9 andar

Curitiba - PR

CEP: 80420-030

E-mail: bianca.zeigelboim @utp.br 